\title{
THE DISTRIBUTION \\ OF MONTIA FONTANA L. (PORTULACACEAE) IN POLAND
}

\author{
Zofia Sotek $^{1}$, AgnieszKa Popiela ${ }^{1}$, Pawet KWIATKOWSKI ${ }^{2}$ \\ ${ }^{1}$ Department of Botany, University of Szczecin \\ Felczaka 3a, 71-412 Szczecin, Poland \\ e-mail: zofia.sotek@sus.univ.szczecin.pl \\ popiela@univ.szczecin.pl \\ ${ }^{2}$ Department of Botany and Plant Physiology, Academy of Agriculture \\ Cybulskiego 32, 50-205 Wroclaw, Poland \\ e-mail: pkwiat@ozi.ar.wroc.pl.
}

(Received: April 10, 2002. Accepted: July 11, 2002)

\begin{abstract}
The distribution of Montia fontana L. in Poland is described, based on accessible data. Maps showing the distribution of M. fontana agg., subsp. amporitana, subsp. chondrosperma are presented, as well as lists of localities with brief descriptions of taxonomic characteristics of the subspecies and their habitats.
\end{abstract}

KEY WORDS: Montia fontana agg., endangered species, distribution, Poland.

\section{INTRODUCTION}

Localities of Montia fontana L., one of the rarest species in Poland, are scattered all over the country (see e.g. Jage 1979; Rothmaler 1990; Lampe 1996). In Central Europe this species is included in so-called "red books", of Brandenburg, Mecklenburg and the Czech Republic among others (Benkert and Klemm 1990; Fukarek 1992, Čeřovský et al. 1999). In Poland M. fontana reaches the eastern limit of its range and it is locally classified as a taxon in direct danger of extinction or extinct (see: Jasiewicz 1981, Żukowski and Jackowiak 1995, Rutkowski 1997, Sotek and Popiela 2001).

The taxonomical revision of Montia fontana was performed by Walters (1953). The author distinguished the following subspecies: subsp. fontana $(=M$. lamprosperma Cham. $=$ M. rivularis auct.), subsp. chondrosperma (Fenzl) S. Walters (= M. minor auct. + M. minor C. C. Gmel. $=M$. verna auct. $=M$. arvensis Wallr.), subsp. amporitana Sennen $(=$ M. hallii (A. Gray) Greene, $=$ M. lusitanica Sampaio, $=M$. fontana subsp. intermedia (Beeby) S. Walters, = M. rivularis auct. mult. + M. rivularis $\mathrm{C}$. C. Gmel.) and subsp. variabilis S. Walters (= M. hallii (A. Gray) Greene var. variabilis (S. Walters) Holub, $=$ M. rivularis auct. mult.). In Poland, the presence of the first three taxa was confirmed (Stengl-Rejthar 1992). The diagnostic features differentiating the four taxa concern seed morphology. In subsp. fontana ripe seeds are smooth and shining; in subsp. chondrosperma - dull and entirely covered with broad ob- tuse tubercles; in subsp. amporitana ripe seeds have 3-4 rows of long, acute tubercles on a keel; and finally in subsp. variabilis - ripe seeds have variably developed broad, low tubercles on a keel.

In the "Distribution Atlas of Vascular Plants in Poland" (ATPOL), the map of Montia fontana agg. is included (Popiela and Sotek 2001). However lists of localities of the taxon and distribution maps for subspiecies of $M$. fontana have not been published up to now. The purpose of this paper is to present the details of the distribution of M. fonta$n a$ subspecies in Poland on the basis of accessible data, and to characterize their typical habitats.

\section{MATERIAL AND METHODS}

We have taken into account revised herbarium materials, the literature and both published and unpublished data. The herbarium material for Montia fontana in Poland is poor. A great part of specimens which could be found in our herbaria have been collected outside Poland. Herbarium data labels are also very often illegible. Seeds in many specimens are lacking, that makes the differentiation of subspecies impossible. Moreover, some authors (especially those publishing before the Second World War) had not determined subspecies of Montia fontana or used not clearly defined synonims. That is why the compilation of reliable maps for all subspecies have not been possible therefore we have decided to make maps for $M$. fontana subsp. 
chondrosperma and $M$. fontana subsp. amporitana, which have been well distinguished and named. The $10 \times 10 \mathrm{~km}$ ATPOL grid square system was used for preparation of maps and for an arrangement of lists of localities (Zając 1978). Acronyms of herbaria follow Mirek et al. (1997). Where at present no settlements exist, the name of the nearest village is given in square brackets. The geographical elements to which the subspecies belong have been determined according to Pawłowska (1972) and also based on the published maps and descriptions of their general distribution (Hultén 1958; Meusel et all. 1965; Jage 1979; Jalas and Suominen 1980; Hultén and Fries 1986; Rothmaler 1990; Lampe 1996).

\section{GENERAL DISTRIBUTION AND HABITATS}

Montia fontana subsp. fontana belongs to a cosmopolitan connective element. It occupies large areas in north-eastern Africa, southern Australia, Tasmania, New Zeland, New Guinea, the Japan Islands, Kamchatka, Sakhalin and in the Atlantic part of North America. In Europe, the taxon occurs mainly in the north and in mountains in only single, isolated sites. It exhibits submediterranean-subatlantic type of distribution reaching the coasts of the White Sea northernmost, and the coasts of the Mediterranean and the Black Sea southernmost.

Montia fontana subsp. chondrosperma, M. fontana subsp. amporitana and M. fontana subsp. variabilis belong to a Mediterranean-holarctic connective element. $M$. fontana subsp. chondrosperma has been noted on the coasts and islands of the Mediterranean Sea and in western Europe. Its northernmost localities have been recorded in Scotland and in southern Sweden (Skandia). In the East the taxon rea- ches Poland, Romania and Bulgaria. The ranges of Montia fontana subsp. amporitana and M. fontana subsp. variabilis are not well known. They are probably restricted to southwestern, central and western Europe, and North America. Single localities of the taxa in the southern hemisphere are most likely the result of human activity.

Montia fontana (with the exception of M. fontana subsp. chondrosperma) occurs mostly in the neighbourhoods of springheads poor in calcium carbonate, mainly in the associations of Cardaminetum amarae (Br.-B1. 1926) Maas 1959 and Chrysosplenietum oppositifolii Oberd. et Philippi 1977. It also grows in stagnant and slowly running waters (e.g. in ditches) and in mountainous springhead communities belonging to Cardamino-Montion Br.-BL. 1925, often in the following associations: Cratoneuro-Saxifragetum aizoides Nordhagen 1936, Mniobryo-Epilobietum hornemannii Nordhagen 1943, Allietum sibirici Šmarda 1950, Swertietum perennis Zlatnik 1928, Montio-Bryetum schleicheri Br.-B1. 1925, Scapanietum paludosae (Müll. 1938) Hadač 1983, Caltho minoris-Philonotidetum (Kästner 1938) Hadač 1983, Philonotido fontanae-Montietum (Br.-B1. 1915) Büker et R. Tx. in Büker 1942 and Diobeloni-Montietum Maas 1959 (Hadač 1983; Oberdorfer 1983; Rothmaler 1990; Philippi and Oberdorfer 1992, Zechmeister and Mucina 1994; Schubert et al. 1995).

$M$. fontana subsp. chondrosperma has been often recorded in the temporarily flooded places on wet arable fields, in communities from the class Isoëto-Nanojuncetea $\mathrm{Br}$.-B1. et R.Tx. 1943, namely in Centunculo-Anthoceretum punctati (Koch 1926) Moor 1936 and Cicendietum filiformis Allorge 1922 (e.g. Moor 1936, Pietsch 1973). M. fontana subsp. amporitana has been reported from Holco gayaniBryetum alpinae Jansen 1999 in southern Portugal (Jansen et Menezes de Sequieira 1999).

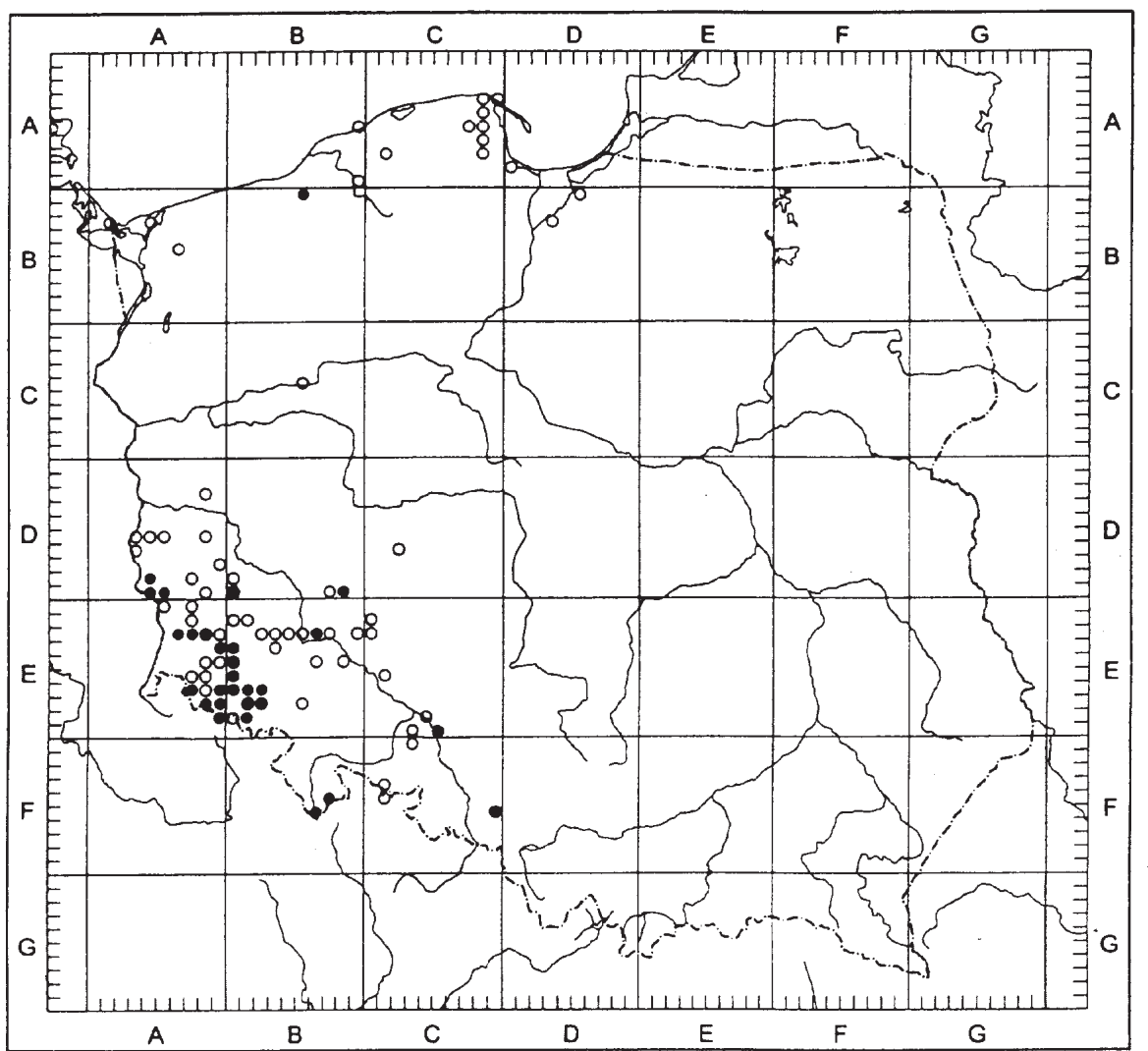

Fig. 1. The distribution of Montia fontana L. agg. in Poland:

- - extant locality; $\bigcirc$ - locality from the literature or herbarium, not confirmed after 1945. 


\section{THE DISTRIBUTION IN POLAND}

All localities of Montia fontana in Poland are restricted to the Sudeten Mts and Foothills, the Silesian Lowland and Pomerania (Fig. 1). A great number of the localities have not been confirmed since 1945. M. fontana subsp. chondro- sperma has been found in south-western Poland, and in Pomerania on isolated sites (Fig. 2), while subsp. amporitana - in the Sudeten Mts and Foothills (Fig. 3). Recently, both subspecies were collected in the Karkonosze Mts, Kaczawskie Mts and Foothills by Kwiatkowski (1997, 2000 and unpublished data), while subsp. amporitana - in the Śnież-
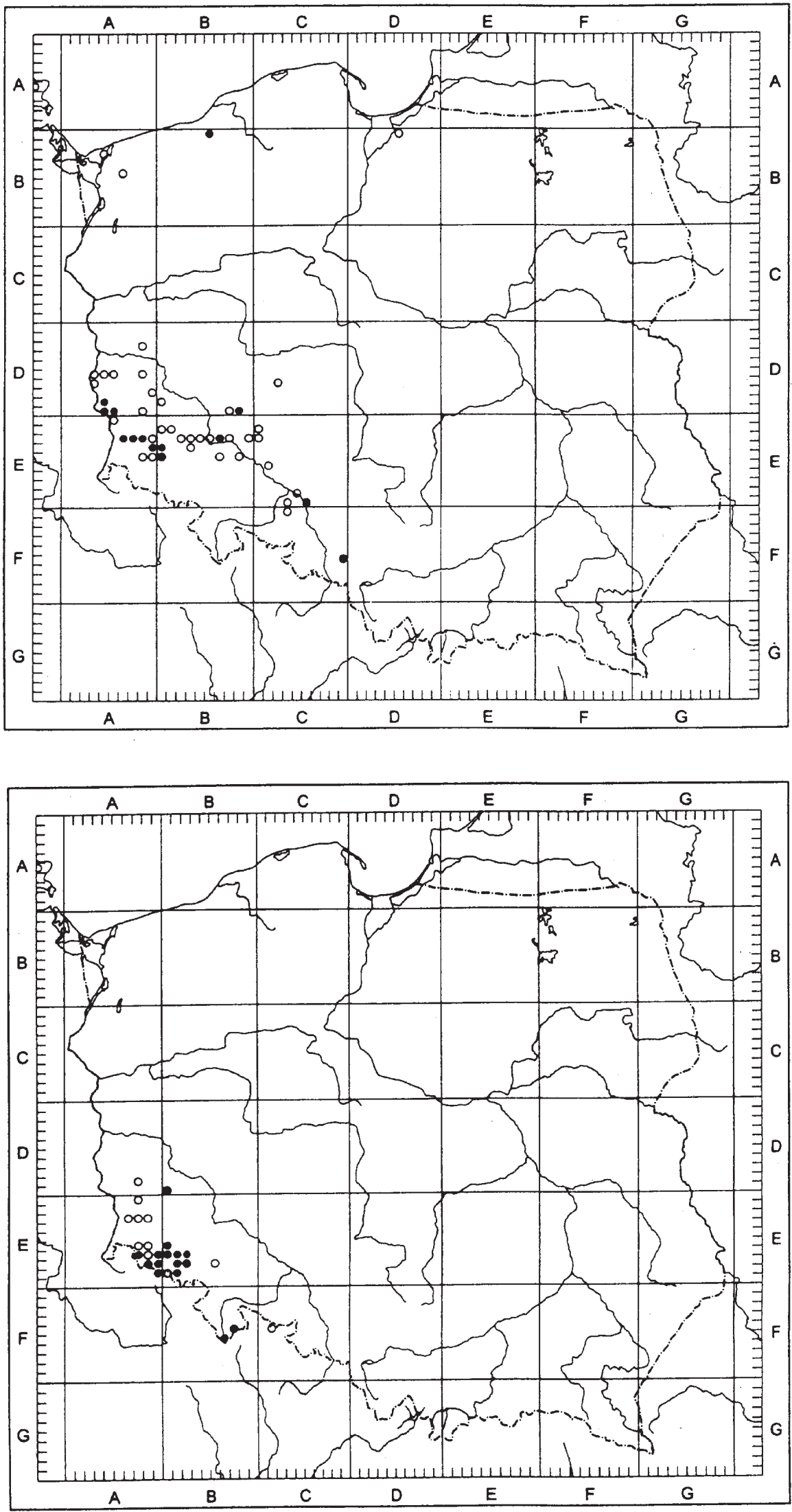

Fig. 2. The distribution of Montia fontana subsp. chondrosperma (Fenzl) S. Walters:

- - extant locality; $\bigcirc$ - locality from the literature or herbarium not confirmed after 1945.
Fig. 3. The distribution of Montia fontana subsp. amporitana Sennen:

- - extant locality; $\bigcirc$ - locality from the literature or herbarium not confirmed after 1945. 
nik Massif by Szeląg (2000). The range of $M$. fontana subsp. fontana is presumably restricted to northern Poland (see e.g. Abromeit et al. 1903).

The phytosociological documentation concerning Montia fontana is insufficient in Poland. Only six relevés of the Diobeloni-Montietum Maas 1959 with participation of $M$. fontana subsp. amporitana were published (Kuczyńska and Berdowski 1976). Literature descriptions and information from herbarium data labels indicate that $M$. fontana occuppies habitats similar to those in Western Europe - springs, swamps, ditches and, in case of subsp. chodrosperma, wet depressions in arable fields (e.g. Uechtritz 1884; Abromeit et al. 1903; Miller 1899; Bock 1908; Müller 1911). Presently, $M$. fontana subsp. amporitana grows in submontane ash marshy forests Carici remotae-Fraxinetum Koch 1926 ex Faber 1936, and in subalpine springheads DiobeloniMontietum Maas 1959, Allietum sibirici Šmarda 1950, Swertietum perennis Zlatnik 1928, whereas subsp. chondrosperma - in lowland alder marshy forests Circaeo-Alnetum Oberd. 1953 and Fraxino-Ulmetum (R.Tx. 1952) Oberd. 1953 and in springheads Chrysosplenietum oppositifolii Oberd. et Philippi 1977 (Kwiatkowski 2000).

In Poland the data reported by Kwiatkowski (1997, 2000, unpublishe data) and Szeląg (2000) are the first information about Montia fontana distribution from 25 years. Presumably the taxon is a fairly common but unnoticeable plant in the Sudeten Mts and Foothills and in the Silesian Lowland. It is probably common also in the Pomerania region, where it had been recorded quite often before 1945 .

\section{LIST OF LOCALITIES IN POLAND}

\section{Montia fontana subsp. amporitana Sennen}

AD grid square: Prov. Lubuskie. 87 - Żagań (Schube 1903, Schalow 1933).

AE grid square: Prov. Lubuskie. 07 - Leszno Górne (Schalow 1933). Prov. Dolnośląskie. 26 - Czerwona Woda (Schalow 1933). 27 - Stary Gaj near Zebrzydowa (Schube 1906, Schalow 1933). 28 - Osieczów (Schube 1903, Schalow 1933). 57 - Mirsk (Schalow 1933). 58 - Lubomierz (Schalow 1932). 67 - Świeradów Zdrój, 1g. E. Kozioł, 1969 (WRSL); Polana Izerska (Głowacki and Kozioł 19741975). 68 - Rębiszów (Schube 1903). 69 - Rybnica (Ciaciura 1965), Wojcieszyce (Ciaciura 1965). 78 - Hala Szrenicka-Kamieńczyk stream (Kuczyńska and Berdowski 1976). 79 - Jagniątków (Winkler 1881, Schalow 1934, Kwiatkowski, unpublished), Szklarska Poręba (Winkler 1881), Cieplice Zdrój (Schalow 1933), Przesieka (Kwiatkowski, unpublished), between Sobieszów and Sosnówka (Schalow 1933). 89 - Karkonosze: Mały Staw Cirque and Wielki Staw Cirque, Wielki Śnieżny Cirque and Mały Snieżny Cirque, Czarny Jagniątkowski Cirque (Kwiatkowski, unpublished), Cirque under Śnieżka (Winkler 1881).

BD grid square: Prov. Lubuskie. 90 - Przemków (Schube 1926, Schalow 1933), Młynów, Karpie (Ciaciura 1971).

BE grid square: Prov. Dolnośląskie. 50 - Janówek, Leśniak, Okole, Chrośnickie Kopy (Czernicka, Ptasia, Kazalnica), Łysa Góra (Kwiatkowski 2000). 60 - Płoszczyna (Kwiatkowski 1997), Ulina (Kwiatkowski 2000). 61 Podgórki, Marciniec, Baraniec, Żeleźniak, Bukowina, Komarno, Radomierz (Kwiatkowski 2000). 62 - Bienwald [Grudno] (Schube 1903), Wapniki (Kwiatkowski 2000). 71
- Marciszów Górny (Schalow 1934), Ciechanowice (Kwiatkowski 2000); Rędzinki, Czarnów, Wielka Kopa, Leszczyniec (Kwiatkowski, unpublished). 72 - Marciszów, Domanów (Kwiatkowski 2000). 75 - Bystrzyca Dolna (Schube 1903), 80 - Karpacz-Wilcza Poręba (Schalow 1933), Ścięgny (Schalow 1933), Kowary (Winkler 1881). 81 - Jarkowice (Kwiatkowski, unpublished), Stara Białka (Ciaciura 1972).

BF grid square: Prov. Dolnośląskie. 47 - Śnieżnik (Szeląg 2000, after Uechtritz), Kletno (Stengl-Rejthar 1992). 56 - Potoczek (Szeląg 2000).

CF grid square: Prov. Opolskie. 41 - Podlesie k. Głuchołazów (Schube 1927).

Montia fontana subsp. chondrosperma (Fenzl) S. Walters AB grid square: Prov. Zachodniopomorskie. 24 - Lake Racze (Piotrowska 1966, after Lucas 1860); 46 - between Golczewo and Moracz (Müller 1911).

AD grid square: Prov. Lubuskie 28 - Podła Góra (Fiek and Pax 1889). 53 - Markosice (Lademann 1938); Brzozów (Lademann 1938). 54 - between Kaniów and Przyborowice (Lademann 1938). 55 - Grabków (Decker 1924). 58 - between Ochla and Jędrzychów (Schube 1913). 63 Suchodół (Decker 1924); Węgliny (Lademann 1938). 79 Kożuchów (Schube 1903). 84 - Chudzowice (Ciaciura 1965). 94 - Piotrów (Serwatka 1964). 95 - Mielno, lg. E. Kozioł, 1972 (WRSL). 98 - Pruszków (Schube 1902).

AE grid square: Prov. Lubuskie. 05 - Gozdnica (Fiek 1887). Prov. Dolnośląskie. 26 - Węgliniec (Mądalski 1960). 27 - Czerna (Schube 1903), Zebrzydowa, lg. E. Kozioł, 1971 (WRSL). 28 - Osieczów, lg. E. Kozioł, 1971 (WRSL). 29 - Chościszowice (Schube 1903). 39 - Żerkowice (Schube 1903); Suszki, Ustronie (Kwiatkowski 2000). 48 - Gradów (Schube 1903). 49 - Płakowice (Schube 1903).

BB grid square: Prov. Zachodniopomorskie. 05 - Lake Lubiatowo (Mądalski 1960).

BD grid square: Prov. Lubuskie. 80 - Żuków (Schube 1903). Prov. Dolnośląskie. 97 - Świniary (Schube 1896); Laskowa (Schube 1902). Prov. Wielkopolskie. 98 - Rawicz (Szulczewski 1951).

BE grid square: Prov. Dolnośląskie. 10 - Patoka (Schube 1903). 11 - Hammerswalde [Chocianów] (Schube 1907). 22 - between Zimna Woda and Wiercień (Schube 1903). 23 - Bieniowice (Schube 1903). 24 - Lisowice (Schube 1903); Golanka (Schube 1903). 25 - Zagórzyce (Schube 1903). 26 - Uskorz Mały (Fiek and Schube 1895); Łososiowice, lg. E. Kozioł, 1970 [WA], (Głowacki 1973, Głowacki and Kozioł 1974-1975); Wołów (Uechtritz 1884); Piotrowice (Schube 1903). 27 - Rościsławice (Schube 1903). 29 - Trzebnica (Schube 1903). 30 - Sędzimirów, Grodziec, Uniejowice (Kwiatkowski 2000). 33 - Stare Piekary (Schube 1903), Kunice (Schube 1903), Pątnów (Schube 1903). 40 - Nowa Wieś Grodziska, Skorzynice, Nowe Łąki (Kwiatkowski 2000). 46 - Gabel [Środa Śl.] (Schube 1907). 48 - between Złotniki and Leśnica (Schube 1903), Leśnica (Schube 1903).

CD grid square: Prov. Wielkopolskie. 62 - Koźmin (Miller 1899).

CE grid square: Prov. Dolnośląskie. 10 - Czeszów (Schube 1903). 20 - Tarnowice (Schube 1903). 51 - Łaźno near Jelcz (Schube 1912). Prov. Opolskie. 84 - Dobrzeń Wlk. (Schube 1903). 93 - Gościejowice (Schube 1903). 95 - Chmielowice (Schube 1903, Michalak 1965). 
CF grid square: Prov. Opolskie. 03 - Brzęczykowice (Schube 1903). Prov. Śląskie 59 - Rudy (Kuczyńska 1974); Rybnickie Lakes (Uechtritz 1863).

DB grid square: Prov. Warmińsko-Mazurskie. 05 - Elbląg (Kalmuss 1884).

Localities of Montia fontana, where subspecies can not be determined

AB grid square: Prov. Zachodniopomorskie. 21 - Świnoujście (Piotrowska 1966, after Ascherson); Świna-Wapnica (Piotrowska 1966, after Leick).

AE grid square: Prov. Dolnośląskie. 17 - Ołobok (Schube 1903).

BA grid square: Prov. Pomorskie. 59 - Ustka (Müller 1911). 99 - Barcino (Müller 1911).

BC grid square: Prov. Wielkopolskie. 45 - Ciszkowo (Bock 1908).

CA grid square: Prov. Pomorskie. 38 - Tupadła (Abromeit et al. 1903). 39 - Władysławowo (Abromeit et al. 1903). 48 - between Władysławowo and Swarzewo (Abromeit et al. 1903). 57 - Rybno (Abromeit et al. 1903). 58 Wejcherowo (Abromeit et al. 1903). 68 - Lake Zawiat (Abromeit et al. 1903). 71 - Warblewo (Müller 1911). 78 Kielno (Abromeit et al. 1903); Lake Marchowo (Herweg 1915).

CF grid square: Prov. Opolskie. 31 - Nielasdorf [Głuchołazy] (Schube 1903).

DA grid square: Prov. Pomorskie. 80 - Zaspa (Abromeit et al. 1903).

DB grid square: Prov. Pomorskie. 23 - Malbork (Abromeit et al. 1903).

\section{ACKNOWLEDGEMENTS}

We would like to thank Prof. Adam Zając for his comments on this manuscript, and Prof. Adam Zając and Dr. Maria Zając for giving us the access to the ATPOL data base.

\section{LITERATURE CITED}

ABROMEIT J., JENTZSCH A., VOGEL G. 1903. Flora von Ostund Westpreussen. I. Samenpflanzen oder Phanerogamen. ix +1248 pp. Preussischen Botanischen Verein zu Königsberg in Preussen, Berlin.

BENKERT D., KLEMM G. 1990. Rote Liste Gefärdete Farnund Blütenpflanzen im Land Brandenburg. 216 pp. Ministerium für Umwelt Naturschutz und Raumordnung des Landes Brandenburg.

BOCK W. 1908. Taschenflora von Bromberg (Das Netzegebiet). xiv +214 pp. Mittler'sche Buchhandlung, Bromberg.

CIACIURA M. 1965. Notatki florystyczne ze Ślaska za rok 1962 [Floristic notes from Silesia for the year 1962]. Opol. Tow. Przyj. Nauk, Zesz. Przyr. 5: 163-170 (in Polish with English summary).

CIACIURA M. 1971. Notatki florystyczne ze Śląska za rok 1966 [Floristic notes from Silesia for the year 1966]. Opol. Tow. Przyj. Nauk, Zesz. Przyr. 11: 71-86 (in Polish with English summary).

CIACIURA M. 1972. Notatki florystyczne ze Śląska za rok 1964 [Floristic notes from Silesia for the year 1964]. Opol. Tow. Przyj. Nauk, Zesz. Przyr. 12: 25-34 (in Polish with English summary)
ČEŘOVSKÝ J., FERÁKOVÁ V., HOLUB J., MAGLOCKÝ Š., PROCHÁZKA F. 1999. Červená Kniha ohrožených a vzácných druhů rostlin a živočichů ČR a SR. 5. Vyšši rostliny. 456 pp. Přiroda a.s., Bratislava.

DECKER P. 1924. Beiträge zur Flora der südlichen Neumark und der östlichen Niederlausitz II. Verh. Bot. Ver. Prov. Brandenburg 66: 86-119.

FIEK E. 1887 [1888]. Resultate der Durchforschung der Schlesischen Phanerogamenflora im Jahre 1887. Jahresber. Schles. Ges. Vaterl. Cultur 65: 309-339.

FIEK E., PAX F. 1889. Resulate der Durchforschung der Schlesischen Phanerogamenflora im Jahre 1888. Jahresber. Schles. Ges. Vaterl. Cultur 66: 174-206.

FIEK E., SCHUBE T. 1895. Resultate der Durchforschung der Schlesischen Phanerogamenflora im Jahre 1894. Jahresber. Schles. Ges. Vaterl. Cultur 73: 92-123.

FUKAREK F. (ed.) 1992. Rote Liste der gefärdete Höheren Pflanzen Mecklenburg-Vorpommerns. 64 pp. GoldschmidtDruck, Schwerin.

GŁOWACKI Z. 1973. Notatki florystyczne z powiatu wołowskiego (Dolny Śląsk). Część III [Floristische Notizen aus dem Kreise Wołów (Niederschlesien). Teil III]. Opol. Tow. Przyj. Nauk, Zesz. Przyr. 13: 41-47 (in Polish with German summary).

GŁOWACKI Z., KOZIOŁ E. 1974-1975. Notatki florystyczne z Dolnego Śląska [Floristische Notizen aus Niederschlesien]. Opol. Tow. Przyj. Nauk, Zesz. Przyr. 14-15: 33-37 (in Polish with German summary).

HADAČ E. 1983. A Survey of Plant Communities of Springs and Mountain Brooks in Czechoslovakia. Folia Geobot. Phytotax. 18: 339-361.

HERWEG O. 1915. Flora der Kreise Neustadt und Putzig in Westpreussen. Ber. Westpreus. Bot.-Zool. Gesell. 35: 85-331.

HULTÉN E. 1958. The amphiatlantic plants and their phytogeographical connections. Kungl. Svenska Vetenskapsakademiens Handlingar, Fjarde Serien. 7 (1): 1-340.

HULTÉN E., FRIES M. 1986. Atlas of North European vascular plants, north of the tropic of Cancer. 1. xviii $+498 \mathrm{pp}$. Koelz Scientific Books, Königsstein.

JAGE H. 1979. Familie Portulacaceae. In: Conert H.J., Hamann U., Schultze-Motel W., Wagenitz G. (eds), Gustav Hegi Illustrierte Flora von Mitteleuropa 3 (2). 1265 pp. Paul Parey, Berlin-Hamburg.

JALAS J., SUOMINEN J. (eds) 1980. Atlas Florae Europaeae. Distribution of vascular plants in Europe. 5. Chenopodiaceae to Basellaceae. Maps 479-668. 119 pp. Suomalaisen Kirjallisuunden Kirjapaino Oy, Helsinki.

JANSEN J., MENEZES DE SEQUEIRA M. 1999. The vegetation of shallow waters and seasonally inundated habitats (Litorelletea and Isoëto-Nanojuncetea) in the higher parts of Serra da Estrela, Portugal. Mitt. Bad. Landesver. Naturkunde Naturschutz. N. F. 2: 449-462.

JASIEWICZ A. 1981. Wykaz gatunków rzadkich i zagrożonych flory polskiej [List of Rare and Endangered Plants fron the Polish Flora]. Fragm. Flor. Geobot. 27: 401-414 (in Polish with English summary).

KALMUSS F. 1884 Die Flora des Elbinger Kreises. Ber. Westpreus. Bot.-Zool. Gesell. 7: 91-159.

KUCZYŃSKA I. 1974. Stosunki geobotaniczne Opolszczyzny. Cz. II. Analiza geograficzna flory. Podział Geobotaniczny [Die geobotanischen Verhältnisse auf dem Gebiet der Woiwodschaft Opole. II. Geographische Analyse der Flora Geobotanische Einteilung]. Acta Univ. Wrat. 198, Prace Bot. 18: 3-114. (in Polish with German summary).

KUCZYŃSKA I., BERDOWSKI W. 1976. Udział Chrysosplenium oppositifolium L. w zbiorowiskach roślinnych Dolnego Śląska [The participation of Chrysosplenium oppositifolium in plant communities of the Lower Silesia]. Acta Univ. Wrat. 303, Prace Bot. 21: 69-86 (in Polish with English summary). 
KWIATKOWSKI P. 1997. Interesujące i rzadkie gatunki roślin naczyniowych Gór Kaczawskich i Pogórza Kaczawskiego. II [Interesting and rare species of vascular plants from Kaczawskie Mountains and Foothills. Part II]. Acta Univ. Wrat. 1936, Prac. Bot. 73: 47-62 (in Polish with English summary).

KWIATKOWSKI P. 2000. Notatki florystyczne z Gór i Pogorza Kaczawskiego (Sudety Zachodnie) [Floristic notes from the Kaczawskie Mts and Kaczawskie Plateau (Western Sudety Mts]. Fragm. Flor. Geobot. Polonica 7: 105-116 (in Polish with English summary).

LADEMANN O. 1938. Beitrage zur Flora der Kreise Guben, Krossen (Oder) und Sorau. Verh. Bot. Ver. Prov. Bradenburg 78: 28-42.

LAMPE M. von 1996. Wuchsform, Wuchsrhythmus und Verbreitung der Arten der Zwergbinsengesellschaften. Diss. Bot. 226: 1-353.

MĄDALSKI J. 1960. Atlas Flory Polskiej i Ziem Ościennych ["The Atlas of flora of Poland and the neigbouring areas"]. 7 (2). p. 31 + 37 Fig. Państwowe Wydawnictwo Naukowe. Warszawa-Wrocław (in Polish).

MEUSEL H., JÄGER E., WEINERT E. 1965. Vergleichende Chorologie der Zentraleuropäischen Flora. 1 (Karten). 258 pp. Gustav Fischer, Jena.

MICHALAK S. 1965. Materiały zielnikowe Muzeum Śląskiego [Herbarium materials of the Opole Museum]. Opol. Tow. Przyj. Nauk, Zesz. Przyr. 5: 29-40 (in Polish with English summary).

MILLER H. 1899. Zur Flora der Gegend von Koschmin. Zeitschrift Bot. Abteilung 5 (3): 76-86.

MIREK Z., MUSIAŁ L., WÓJCICKI J. 1997. Polish herbaria. Polish Bot. Stud. Guidebook Series 18: 3-116.

MÜLLER W. 1911. Flora von Pommern. vi + 1-376 pp. Johns. Burmeister Buchhandlung, Szczecin.

MOOR M. 1936. Zur Soziologie der Isoëtalia. Beitr. Geobot. Landesaufn. Schweiz 20: 1-148.

OBERDORFER E. 1983 (ed.). Pflanzensoziologische Excursionsflora. 1051 pp. E. Ulmer Verlag, Stuttgart.

PAWŁOWSKA S. 1972. Charakterystyka statystyczna i elementy flory polskiej ["Statistical characterization and elements of the Polish flora"]. - In: W. SZAFER and K. ZARZYCKI (eds), Szata roślinna Polski ["The vegetation of Poland"]. 1, pp. 129206. Państwowe Wydawnictwo Naukowe, Warszawa (in Polish).

PHILIPPI G., OBERDORFER E. 1992. Klasse: Montio-Cardaminetea, In: E.OBERFORFER (ed.), Süddeutsche Pflanzengesellsachaften. Teil I. Fels-, und Mauergesellschaften, alpine Fluren, Wasser-, Verlandungs-, und Moorgesellschaften. G. Fischer Verlag Jena-Stuttgart-New York, pp. 199-213.

PIETSCH W. 1973. Beitrag zur Gliederung der europäischen Zwergbinsengesellschaften (Isoëto-Nanojuncetea Br.-Bl. et Tx. 1943). Vegetatio 28: 401-438.

PIOTROWSKA H. 1966. Rośliny naczyniowe wysp Wolina i południowo-wschodniego Uznamu ["Vascular plants of the Wolin island and the south-northern part of the Uznam island"]. Pr. Komis. Biol. Pozn. Tow. Przyj. Nauk. 30 (4): 1-283 (in Polish with English summary).

POPIELA A., SOTEK Z. Montia fontana. 2001. In: A. Zając, M. Zając (eds). Distribution Atlas of vascular plants in Poland (ATPOL). Nakładem Pracowni Chorologii Komputerowej Instytutu Botaniki Uniwersytetu Jagiellońskiego, Kraków.

ROTHMALER W. 1990. Exkursionsflora von Deutschland. 8 Aufl. 4 (Kritischer Band). 811 pp. Volk und Wissen Verlag $\mathrm{GmbH}$, Berlin.

RUTKOWSKI L. 1997. Rośliny naczyniowe - Tracheophyta [Vascular plants - Tracheophyta"]. In: Buszko J., Kasprzyk K., Pawlikowski T., Przystalski A., Rutkowski L. (eds), Czerwona lista roślin i zwierząt ginących i zagrożonych w regionie kujawsko-pomorskim [Red list of endangered plants and animals of Kujavian-Pomeranian Region]. Acta Univ. Nicolai
Copernici, Biol. 53, Supl. (Nauki Mat-przyr. 98): 9-20 (in Polish with English summary).

SCHALOW E. 1932. Die Ergebnisse der Durchforschung der Schlesischen Gefässpflanzenwelt im Jahre 1931. Jahresb. Schles. Ges. Vaterl. Cult. 104: 92-112.

SCHALOW E. 1933. Ergebnisse der Durchforschung der Schlesischen Gefässpflanzenwelt im Jahre 1932. Jahresb. Schles. Ges. Vaterl. Cult. 105: 154-173.

SCHALOW E. 1934. Ergebnisse der Durchforschung der Schlesischen Gefässpflanzenwelt im Jahre 1933. Jahresb. Schles. Ges. Vaterl. Cult. 106: 140-156.

SCHUBE T. 1896 [1897]. Ergebnisse der Durchforschung der Schlesischen Gefässpflanzenwelt im Jahre 1896. Jahresber. Schles. Ges. Vaterl. Cultur 74: 39-64.

SCHUBE T. 1902. Ergebnisse der Durchforschung der Schlesischen Gefässpflanzenwelt im Jahre 1902. Jahresber. Schles. Ges. Vaterl. Cultur 80: 33-56.

SCHUBE T. 1903. Die Verbreitung der Gefässpflanzen in Schlesischen preussischen und österreichischen Anteils. ss. iv + 361. R. Nischkowsky, Breslau.

SCHUBE T. 1906. Ergebnisse der Durchforschung der Schlesischen Gefässpflanzen im Jahre 1905. Jahresb. Schles. Ges. Vaterl. Cult. 83: 75-95.

SCHUBE T. 1907. Ergebnisse der Durchforschung der Schlesischen Gefässpflanzen im Jahre 1906. Jahresb. Schles. Ges. Vaterl. Cult. 84: 68-89.

SCHUBE T. 1912. Ergebnisse der Durchforschung der Schlesischen Gefässpflanzen im Jahre 1911. Jahresb. Schles. Ges. Vaterl. Cult. 89: 57-70.

SCHUBE T. 1913. Ergebnisse der Durchforschung der Schlesischen Gefässpflanzen im Jahre 1912. Jahresb. Schles. Ges. Vaterl. Cult. 90: 92-103.

SCHUBE T. 1926. Ergebnisse der Durchforschung der Schlesischen Gefässpflanzen im Jahre 1925. Jahresb. Schles. Ges. Vaterl. Cult. 98: 9-15.

SCHUBE T. 1927. Ergebnisse der Durchforschung der Schlesischen Gefässpflanzen im Jahre 1926. Jahresb. Schles. Ges. Vaterl. Cult. 99: 24-30.

SCHUBERT R., HILBIG W., KLOTZ S. 1995. Bestimmungsbuch der Pflanzengeselschaften Mittel- und Nordostdeutschlands. 403 pp. Gustav Fischer Verlag, Jena-Stuttgart

SERWATKA J. 1964. Nowe stanowiska rzadszych gatunków roślin naczyniowych na Śląsku [New locations of more rare species of vascular plants in Silesia]. Opol. Tow. Przyj. Nauk, Zesz. Przyr. 4: 115-120 (in Polish with English summary).

SOTEK Z., POPIELA A. 2001. Montia fontana L. In: KAŹMIERCZAKOWA R., ZARZYCKI K. (eds), Polska Czerwona Księga Roślin. Paprotniki i rośliny kwiatowe. [Polish Red Data Book of Plants. Pteridophytes and flowering plants]. 92-93 pp. Instytut Botaniki im. W. Szafera, Polska Akademia Nauk, Kraków (in Polish with English summary).

STENGL-REJTHAR A. 1992. Portulacaceae. In: Jasiewicz A. (ed.), Flora Polski. 3, pp. 224-226. Instytut Botaniki im. W. Szafera, Polska Akademia Nauk, Kraków (in Polish).

SZELĄG Z. 2000. Rośliny naczyniowe Masywu Śnieznika i Gór Bialskich [Vascular plants of the Śnieżnik Massif and the Góry Bialskie]. Fragm. Flor. Geobot. Polonica Suppl. 3: 3-225 (in Polish with English summary).

SZULCZEWSKI J.W. 1951. Wykaz roślin naczyniowych w Wielkopolsce dotąd stwierdzonych ["A list of vascular plants found up to the present from the Wielkopolska region"]. Pr. Komis. Biol. Pozn. Tow. Przyj. Nauk Supl. 12 (6): 1-128 (in Polish).

UECHTRITZ R. 1863. Nachträge zur Flora von Schlesien (II) Verh. Bot. Ver. Prov. Brandenburg. 5: 118-157.

UECHTRITZ R. 1884. Resultate der Durschforschung der Schlesischen Phanerogamenflora im Jahre 1883. Jahresber. Schles. Ges. Vaterl. Cultur 61: 249-300.

WALTERS S.M. 1953. Montia fontana L. Watsonia 3: 1-6. 
WINKLER W. 1881. Flora des Riesen- und Isergebirgen. viii + 234 pp. E. Gruhn, Warmbrunn.

ZAJĄC A. 1978. Atlas of distribution of vascular plants in Poland (ATPOL). Taxon 27: 481-484.

ZECHMEISTER H., MUCINA L. 1994. Vegetation of European Springs: High-rank syntaxa of the Montio-Cardaminetea. J. Veget. Sci. 5: 385-402.

ŻUKOWSKI W., JACKOWIAK W. 1995. Lista roślin naczyniowych ginących i zagrożonych na Pomorzu Zachodnim i w Wielkopolsce [List of endangered and threatened vascular plants in Western Pomerania and Wielkopolska (Great Poland)]. - In: W. Żukowski and B. Jackowiak (eds), Ginące i zagrożone rośliny naczyniowe Pomorza Zachodniego i Wielkopolski [Endangered and threatened vascular plants in Western Pomerania and Wielkopolska]. - Prace Zakł. Taks. Rośl. Uniw. A. Mickiewicza w Poznaniu 3: 9-92 (in Polish and English).

\section{ROZMIESZCZENIE MONTIA FONTANA L. (PORTULACACEAE) W POLSCE}

\section{STRESZCZENIE}

Na podstawie danych z literatury, zielnikowych oraz własnych badań terenowych przedstawiono aktualne rozmieszczenie Montia fontana agg., M. fontana subsp. amporitana i M. fontana subsp. chondrosperma w Polsce. Wyniki zaprezentowano w postaci wykazu stanowisk i map z zasostosowaniem metody ATPOL. Ponadto przedyskutowano problemy taksonomiczne oraz scharakteryzowano ogólne warunki siedliskowe i stosunki fitocenotyczne badanych taksonów.

SŁOWA KLUCZOWE: Montia fontana agg., gatunki zagrożone, rozmieszczenie, Polska. 